\title{
A Theory of Farsightedness in Committee Games
}

\author{
Alphonse Fodouop Fotso, ${ }^{1}$ Joseph Armel Momo Kenfack, ${ }^{1}$ and Bertrand Tchantcho ${ }^{1,2}$ \\ ${ }^{1}$ MASS Laboratory, The University of Yaoundé 1, P.O. Box 47, Yaoundé, Cameroon \\ ${ }^{2}$ THEMA Laboratory, University of Cergy Pontoise, 33 Boulevard du Port, 95011 Cergy-Pontoise Cedex, France
}

Correspondence should be addressed to Bertrand Tchantcho; btchantcho@yahoo.fr

Received 11 October 2013; Revised 17 December 2013; Accepted 31 December 2013; Published 3 April 2014

Academic Editor: Jacqueline Morgan

Copyright (c) 2014 Alphonse Fodouop Fotso et al. This is an open access article distributed under the Creative Commons Attribution License, which permits unrestricted use, distribution, and reproduction in any medium, provided the original work is properly cited.

\begin{abstract}
We study the committee decision making process using game theory. A committee here refers to any group of people who have to select one option from a given set of alternatives under a specified rule. Shenoy (1980) introduced two solution concepts, namely, the one-core and a version of bargaining set for committee games. Shortcomings of these solutions concepts are raised and discussed in this paper. These shortcomings are resolved by introducing two new solutions concepts: the farsighted one-core and the bargaining set revised, inspired by an idea of farsightedness initially defined by Rubinstein (1980). It is shown that the farsighted one-core is always non-empty and is better than the one-core. In a well-specified sense, the bargaining set revised is also better than the bargaining set as defined by Shenoy (1980) and it is always non-empty for simple committee games with linear preferences. Other attractive properties are also proved.
\end{abstract}

\section{Introduction}

Our game model is the one considered by Shenoy [1], committee game that generalizes the voting model introduced by von Neumann and Morgenstern [2] under the name of simple game. A committee game consists in any finite group $N$ of persons who have to pick one option from the finite given set of outcomes $A$ through a voting rule $v$ by which the committee arrives at a decision. The rule $v$ is designed such that the decision of the committee will consist of a unique outcome. Any player is allowed to suggest any alternative for consideration by the committee and players get their payoffs only when the committee has made a decision. In such a social choice context, the question generally asked is how a player should behave or should vote when solicited to join a coalition in order to decide over a status quo. Another relevant issue is to determine what could be a suitable choice of a given player $i$ if he is given the opportunity to introduce a motion.

The core is a solution concept in which any player is recommended to vote for $x$ against $y$ whenever he strictly prefers $x$ to $y$ (i.e., $\left.u_{i}(x)>u_{i}(y)\right)\left(u_{i}\right.$ is the player utility function; instead of considering utilities vectors one could consider that each member of the committee has a preference relation which is a weak order on the set of all outcomes, thus yielding a preference profile) if $x$ is opposed to $y$. Furthermore, a committee member should propose an outcome $x$ if it is the (or one of his) best element in the core. An outcome $x$ belongs to the core if it is undominated, that is, there does not exist another outcome $y$, a coalition $S$ powerful on $y$ and all members of which are strictly better off at $y$ than at $x$. This behavioral pattern of the core has been criticized by Shenoy [1] who argued that a player who is making a proposal does not cooperate in any effort to dominate the proposal. In other words such a player cares about undominated outcomes via coalitions not containing him and picks only maximal ones. This yields the definition of the one-core. Unfortunately, the one-core might be empty even if players' preferences are strict. Furthermore, we show through a simple example of 3-person committee game that players are not farsighted while making proposals under one-core behavioral pattern. We resolve this lack of farsightedness by introducing another solution concept, the farsighted one-core for committee games. It is shown that this new solution concept is better than the one-core. Moreover, when preferences are strict orders, the farsighted one-core of any simple committee game is always nonempty. 
Another contribution of Shenoy [1] is the introduction of a bargaining set for committee games. The concept of bargaining set was first introduced by Aumann and Maschler [3] in the context of games with side payments. They defined several kinds of bargaining sets. These sets were generalized for games without side payments and studied by Peleg [4], Billera [5, 6], D’Aspremont [7], and Asscher [8]. Since then, several other modifications of the bargaining set have been studied in different contexts. The bargaining set introduced by Shenoy [1] is relevant for committee games. Like other bargaining sets, it is based on objections and counter-objections. A proposal is said to be $M$-stable if every objection has a counter-objection. Let $\widehat{M}$ be the set of all $M$-stable proposals. A $M$-stable proposal $(i, x)$ is said to be maximal if $x$ is at least as good as $y$ for all $y$ such that $(i, y) \in \widehat{M}$. The bargaining set $\mathscr{M}$ is the set of all maximal $M$-stable proposals. The Shenoy bargaining set can be empty. We revise this solution concept by introducing the revised Shenoy bargaining set which is proved to be better than the latter. Moreover, when indifference is not allowed in individual rankings, the farsighted bargaining set of any simple committee game introduced herein is nonempty.

The rest of the paper is organized as follows. Section 2 is devoted to the model and preliminaries. In Section 3, we define the farsighted one-core and we prove that it is better than the one-core. Moreover, we prove that the farsighted one-core of any committee game is always nonempty, provided that individual preferences are linear orders. A comparison of the farsighted one-core with other solution concepts is conducted therein. In Section 4, we revise Shenoy bargaining set by reconsidering the definition of objection and counter-objection. It is shown that the new bargaining set improves on the latter defined by Shenoy for committee games. Conclusion, which is Section 5, ends the paper.

\section{The Setting and Preliminaries}

2.1. The Model. Throughout the paper the set of players, that is, the committee, is denoted by $N=\{1,2, \ldots, n\}$; the finite set of candidates or outcomes is $A$. It is assumed that $A$ has at least three elements. Nonempty subsets of $N$ are called coalitions and the set of all coalitions of $N$ is denoted by $2^{N} ;|T|$ stands for the cardinality of any set $T$. The preference relation of any player on $A$ is a weak order (reflexive and transitive relation). If $\geq_{i}$ denotes the preference of player $i$ and $a$ and $b$ are two outcomes, $a \geq_{i} b$ means that, according to $i, a$ is at least as good as $b . a>_{i} b$ means that $i$ strictly prefers $a$ to $b$ and $a \sim_{i} b$ means that $i$ is indifferent between $a$ and $b$. A profile $R$ is a collection of individual preferences, $R=\left(R^{i}\right)_{i \in N}$.

The rules by which the committee members arrive at a decision are called the characteristic function which is a mapping $v: 2^{N} \rightarrow \mathscr{P}(A)$, where $\mathscr{P}(A)$ designates the set of subsets of $A$. For any coalition $S, v(S)$ denotes the subset of outcomes that coalition $S$ can realize if the decision is unanimous in $S$. This means that, at any time, an outcome $x$ becomes the final outcome of the game, whenever a coalition $S$ such that $x \in v(S)$ asks for the adoption of $x$. It is assumed that $v$ satisfies the following conditions:

(C1) for all $S_{1}, S_{2} \in 2^{N}, S_{1} \subseteq S_{2} \Rightarrow v\left(S_{1}\right) \subseteq v\left(S_{2}\right)$,

(C2) $v(N)=A$,

$$
\begin{gathered}
\forall S_{1}, S_{2} \in 2^{N}, \\
\begin{array}{c}
S_{1} \cap S_{2}=\emptyset \\
\left(S_{1}\right) \neq \emptyset, v\left(S_{2}\right) \neq \emptyset
\end{array} \Longrightarrow v\left(S_{1}\right)=v\left(S_{2}\right), \quad\left|v\left(S_{1}\right)\right|=1 .
\end{gathered}
$$

Condition $(\mathrm{C} 1)$ is the well-known monotonicity condition; (C2) means that the whole committee members can enforce any alternative. Condition (C3) ensures that the committee decision consists of at most one outcome. The tuple $\Gamma=(N, A, v, R)$ is called an (ordinal) $n$-person committee game. $R$ could also be replaced with a utility vector $u=\left(u^{i}\right)$, where $u^{i}: A \rightarrow \mathbb{R}$ denotes the real-valued ordinal utility function of player $i$. Here, utility is assumed to be nontransferable and interpersonal comparison of utilities has no meaning.

The committee aims at choosing one option from the set $A$ of outcomes. The members of the committee are considered to be situated in one room. As in Shenoy [1], we are primarily concerned with small committees that arrive at a decision after lengthy deliberations. In this respect the model considered here differs fundamentally from the theory of elections where the decision makers (the players) are numerous and spread out extensively. Let us remark that the committee game model fits very well into the more general model of social environments. A social environment is described by a tuple $\left(N, Z,\left(\rightarrow{ }_{S}\right)_{S \in 2^{N}},(\preceq)_{i \in N}\right)$, where $N$ is the set of players, $Z$ the set of outcomes, and $\left\{\rightarrow_{S}\right\}$ are effectiveness relations defined on $Z$. The relation $\rightarrow_{s}$ represents what coalition $S$ can do; $a \rightarrow{ }_{s} b$ means that, if $a$ is status quo, $S$ can make $b$ the new status quo. It does not mean that $S$ can enforce $b$ no matter what anyone else does. After the move of $S$ to $b$ another coalition $T$ might move to $c$ and so on. Social environments have been considered in many works in the literature including Chwe [9], Xue $[10,11]$, Suziki and Muto [12], Béal et al [13], and Kenfack and Tchantcho [14]. In a committee game, if $b \in v(S)$ then $S$ can enforce $b$. Note on the other hand that committee games generalize the model of simple games. In this respect, a committee game $\Gamma=(N, A, v, R)$ is said to be simple if $\forall S \in 2^{N}, v(S)=\emptyset$, or $v(S)=A$. If $v(S)=\emptyset$ then $S$ is a losing coalition and if $v(S)=A, S$ is a winning coalition. In a simple committee game, a coalition is a minimal winning coalition if it is a winning coalition and if every proper subset of $S$ is a losing coalition. In such a game, the set of winning coalitions is denoted by $\mathscr{W}$ while the set of minimal winning is denoted by $\mathscr{W}^{m}$. Player $i$ is said to be a dummy player if $i \notin \bigcup_{S \in \mathscr{W}^{m}} S$.

It is assumed that a particular outcome say $a_{0}$ is the initial status quo. $a_{0}$ will be the decision of the committee if it cannot 
agree on any other outcome or if it specifically picks $a_{0}$ to be the final decision. There is no agenda (a linear order on A specifying in which order candidates are confronted) and any member $i$ of the committee is allowed to suggest any alternative $x$ at any time for consideration by the committee in the form of a proposal $(i, x)$. The game ends at an option $a^{*}$ such that there is no credible contestation. Such an option is said to be stable.

2.2. Recall of Dominance Relations. We give below the definition of 1-dominance that is suitable for committee games. This is a transposition for committee games of the dominance introduced by Rubinstein [15] for social decision systems. According to 1-dominance, a player $i$ should participate in the elimination of motion $a$ for $b$ only if any subsequent deviation from $b$ to $c$ by another coalition $L$ does not worsen the utility of $i$ relative to $a$.

Definition 1. Let $\Gamma=\left(N, A, v,\left(\succeq_{i}\right)_{i \in N}\right)$ be a committee game, $a, b \in A$, and $S$ a coalition.

(1) $a$ 1-dominates $b$ via $S$ denoted by $a 1-\operatorname{dom}_{S} b$ if $a \in$ $v(S)$, for all $i \in S, a \succ_{i} b$, and for all $c \in A, c \operatorname{dom} a \Rightarrow$ $c \succeq_{i} b$ for all $i \in S$.

(2) $a$ 1-dominates $b$ denoted by $a$ 1-dom $b$ if there exists a coalition $S \in 2^{N}$ such that $a 1-\operatorname{dom}_{S} b$.

According to the 1-dominance, a coalition will refrain from blocking an alternative, say $b$, by voting for $a$, if $a$ may be blocked later on by another coalition voting for, say, $c$ against $a$, if it turns out that $c$ is worse than $a$ for some of its members. It is obvious that with respect to behavioral purposes, the 1-dominance improves the (classical) dominance recalled below.

Definition 2. Let $\Gamma=\left(N, A, v,\left(\succeq_{i}\right)_{i \in N}\right)$ be a committee game, $a, b \in A$.

(1) $a$ dominates $b$ via $S$ denoted by $a \operatorname{dom}_{S} b$ if $a \in v(S)$ and for all $i \in S, a>_{i} b$;

(2) $a$ dominates $b$ denoted by $a \operatorname{dom} b$ if there exists $S \in$ $2^{N}$ such that $a \operatorname{dom}_{S} b$.

It follows from this definition that the rational behavior underlying the core prescribes that a player should vote for an alternative $a$ against another alternative $b$ whenever he prefers $a$ to $b$.

As a comparison between these two dominances, one could verify that for any $a, b \in A$, if $a 1$-dom $b$ then $a$ dominates $b$ but the converse is not true. In the next section, we will use the 1-dominance to build our first solution concept: the farsighted one-core.

\section{The Farsighted One-Core of a Committee Game}

We introduce this section with the definition of the farsighted one-core, based on the behavioral considerations captured by the 1-dominance relation. Let us denote by
$P=\{(i, x): i \in N, x \in A\}$ the set of all proposals,

$\widehat{S}^{i}=\{(i, x) \in P: x$ is not 1 -dominated via a coalition $S \subseteq N \backslash\{i\}\}$

$S^{i}=\left\{(i, x) \in \widehat{S}^{i}: x \succeq_{i} y\right.$ for all $\left.(i, y) \in \widehat{S}^{i}\right\}$.

$\widehat{S}^{i}$ represents the set of proposals made by $i$ that are not 1-dominated assuming player $i$ 's noncooperation in any effort to 1-dominate his proposal and $S^{i}$ represents the maximal proposals in the set $\widehat{S}^{i}$.

Definition 3. Let $\Gamma=\left(N, A, v,\left(\succeq_{i}\right)_{i \in N}\right)$ be a committee game. The farsighted one-core of $\Gamma$ denoted by $\mathscr{F}(\Gamma)$ is defined by $\mathscr{F}(\Gamma)=\bigcup_{i \in N} S^{i}$.

Intuitively, the farsighted one-core consists of all (maximal) proposals which are not 1-dominated assuming that the player who makes the proposal does not cooperate in any effort to 1-dominate the proposal. For obvious reasons, assuming all proposals in $\widehat{S}^{i}$ to be equally stable, player $i$ picks only the maximal ones.

Before giving some properties of the farsighted one-core, we shall prove through a simple example that the farsighted one-core overcomes a myopic shortcoming observed in the Shenoy one-core. Before that, let us recall the definition of the one-core as introduced by Shenoy [1].

The core was initially studied explicitly by Gillies [16] and Shapley [17] for transferable utilities games. It is defined as follows.

Definition 4. Let $\Gamma=\left(N, A, v,\left(>_{i}\right)_{i \in N}\right)$ be a committee game. The core of $\Gamma$ is the set of all undominated outcomes; that is, $C(\Gamma)=\{b \in A: \operatorname{not}(\exists a \in A, a \operatorname{dom} b)\}$.

With respect to the core behavioral pattern, a member $i$ of the committee to whom it is given the opportunity to make a proposal should propose the candidate of the core which guarantees a maximal satisfaction. The main shortcoming of the core is its existence, many interesting committee games have empty cores.

The one-core is a solution concept introduced by Shenoy [1] that results from a small modification in the definition of the core. The modification is motivated by behavioral considerations. Indeed, being in the core means not being dominated. The modification provided by Shenoy [1] is that it is better for such a player to propose, instead of that core candidate, a (maximal) proposal which is not dominated assuming that the player himself does not cooperate in any effort to dominate the proposal. Formally, for each $i \in N$, define the following.

$\widehat{C}^{i}=\{(i, x) \in P: x$ is not dominated via any $S \subseteq N \backslash$ $\{i\}\}$, the set of proposals made by $i$ that are undominated assuming player $i$ 's noncooperation in any effort to dominate his proposal, and $C^{i}=\left\{(i, x) \in \widehat{C}^{i}: x \geq_{i} y\right.$ for all $\left.(i, y) \in \widehat{C}^{i}\right\}$, the set of maximal (best) proposals in $\widehat{C}^{i}$.

Definition 5. Let $\Gamma=\left(N, A, v,\left(\succeq_{i}\right)_{i \in N}\right)$ be a committee game. The one-core of $\Gamma$ denoted by $\mathscr{C}_{1}(\Gamma)$ is given by $\mathscr{C}_{1}(\Gamma)=$ $\bigcup_{i \in N} C^{i}$. 


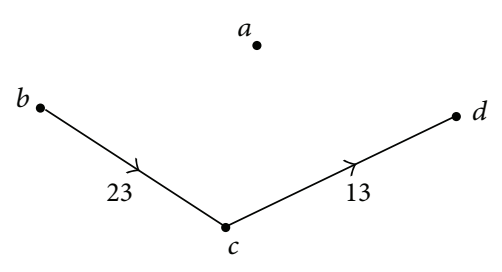

FIGURE 1: Domination graph of Example 6.

The one-core consists of all (maximal) proposals which are undominated assuming that the player who makes the proposal does not cooperate in any effort to dominate the proposal.

Example 6. Let us consider the following 3-player committee game $\Gamma=\left(N, A, v,\left(>_{i}\right)_{i \in N}\right)$, where $N=\{1,2,3\}, A=$ $\{a, b, c, d\}, v(1)=v(2)=v(3)=v(12)=\emptyset, v(13)=\{d\}$, $v(23)=\{c\}, v(123)=A$, and the profile $\left(>_{i}\right)=($ badc, cbad , $d a c b)$.

A simple computation gives $\widehat{S}^{1}=\{(1, b)\}, \widehat{S}^{2}=\{(2, b)\}$, $\widehat{S}^{3}=\{(3, d)\}$, and thus $\mathscr{F}(\Gamma)=\{(1, b),(2, b),(3, d)\}$. As well the core is $\{a, d\}$ and the one-core $\mathscr{C}_{1}(\Gamma)=\{(1, a) ;(2, b)$; $(3, d)\}$, meaning that, if it is given to 1 the opportunity to make a proposal, he shall propose candidate $a$. This happens indeed because $\widehat{C}^{1}=\{(1, a),(1, c),(1, d)\}$ and $a$ is the best of the three proposals, according to 1 . However, we claim that proposing $a$ is not the best strategy for player 1 as we explain in Figure 1. Assume that player 1 proposes $b$.

(i) If $c$ is proposed (either by 2 or by 3 ), then both $c$ and $b$ will be put to vote with the players voting for one of the two motions. It is not in the interest of 2 to vote for $c$. Indeed, if $c$ becomes the new status quo 1 and 3 will certainly enforce the adoption of $d$ as the final outcome. But $d$ is the worst candidate for 2 . Thus even if $c$ is opposed to $b, c$ will not win.

(ii) If $d$ is proposed (by 3 ), then it is obvious that it will be eliminated since the only coalition able to enforce $d$ is 13 and player 1 , who made the initial proposal $b$, will not vote for $d$; hence $b$ will not be defeated!

(iii) For the same reason, if $a$ is proposed it will be defeated.

Finally, as we can see, if 1 proposes $b$, then $b$ will be elected. This shows that if 1 is given the possibility to propose it will be better for him to propose $b$ instead of $a$, as recommended by the one-core.

Thanks to Example 6 above, it is obvious that neither the farsighted one-core includes the one-core nor the one-core includes the farsighted one-core.

In the following result, we prove that if $(i, x)$ is in the farsighted one-core, then $x$ is better than whatever outcome $y$ such that $(i, y)$ belongs to the one-core.

Proposition 7. Let $\Gamma=\left(N, A, v,\left(\succeq_{i}\right)_{i \in N}\right.$ be a committee game. Then, for all $i \in N$, for all $x \in A,(i, x) \in \mathscr{F}(\Gamma) \Rightarrow x \geq_{i} y$ for all $y$ such that $(i, y) \in \mathscr{C}_{1}(\Gamma)$.
Proof. Let $i \in N$ and $x \in A$ such that $(i, x) \in \mathscr{F}(\Gamma)$. Let $y \in A$ such that $(i, y) \in \mathscr{C}_{1}(\Gamma)$. Since $(i, y) \in \mathscr{C}_{1}(\Gamma)$, then $(i, y) \in \widehat{C}^{i}$ and this means that $y$ is undominated via $S$ for each $S \subseteq N \backslash\{i\}$. This latter implies $y$ is undominated (with respect to 1 -dom) via $S$ for each $S \subseteq N \backslash\{i\}$ : thus, $(i, y) \in \widehat{S}^{i}$. Moreover, $(i, x) \in \mathscr{F}(\Gamma)$ implies $(i, x) \in S^{i}$; that is, $x \geq_{i} y$.

The Condorcet solution was first defined by Condorcet [18] and rediscovered independently by Dodgson [19]. It is defined as follows.

Definition 8. Let $\Gamma$ be a committee game. A Condorcet solution of $\Gamma$ is any $a \in A$ that dominates every other outcome in $A$; that is, for all $b \in A \backslash\{a\}, a \operatorname{dom} b$.

It is well known that if a Condorcet solution exists, it is unique. Shenoy [1] showed that if in $\Gamma$ the Condorcet solution exists, say $\alpha$, then $\mathscr{C}_{1}(\Gamma)=\{(1, \alpha) ;(2, \alpha) ; \ldots ;(n, \alpha)\}$.

We show in the following result that if the Condorcet solution exists, then, with respect to the farsighted one-core, every player should propose it.

Proposition 9. Let $\Gamma$ be a committee game such that the Condorcet solution $\alpha$ exists. Then the farsighted one-core is given by $\mathscr{F}(\Gamma)=\{(1, \alpha),(2, \alpha), \ldots,(n, \alpha)\}$.

Proof. Let $\Gamma$ be a committee game such that the Condorcet solution $\alpha$ exists. Then for each player $i \in N, \alpha$ is undominated with respect to the 1-dominance relation via any coalition $S \subseteq N \backslash\{i\}$. Hence, $(i, \alpha) \in \widehat{S}^{i}$ for each player $i \in N$. In addition, for each player $i$ and for each proposal $(i, x)$ with $x \neq \alpha$, if $(i, x) \in \widehat{S}^{i}$, then $\alpha>_{i} x$.

Indeed, assume for some $i \in N$ and $x \in A$ that non $\left(\alpha>_{i} x\right)$. That is, $x \geqslant_{i} \alpha$, since preference relations are weak orders. Then, $\alpha$ being a Condorcet solution implies that there exists $T \subseteq N$ such that $\alpha 1-\operatorname{dom}_{T} x$ and for each player $j \in T$, $\alpha \succ_{j} x$. Therefore, there exists a coalition $T \subseteq N$ such that $\alpha 1$ $\operatorname{dom}_{T} x$ and $i \notin T$. That is, $(i, x) \notin \widehat{S}^{i}$. Hence, $S^{i}=\{(i, \alpha)\}$ for each $i \in N$, and thus $\mathscr{F}(\Gamma)=\{(1, \alpha),(2, \alpha), \ldots,(n, \alpha)\}$.

An important solution concept for social environments in general and committee games in particular is the Chwe [9] largest consistent set. It describes situations where deviating coalitions anticipate the ultimate consequences of their initial move.

Definition 10. Let $\Gamma=\left(N, A, v,\left(\succeq_{i}\right)_{i \in N}\right)$ be a committee game.

(1) Given $a, b \in A, b$ indirectly dominates $a$ (denoted by $b \gg a$ ) if there exists a sequence of outcomes $a=a^{0}, a^{1}, \ldots, a^{p}=b$, and a sequence of coalitions $S^{1}, \ldots, S^{p} \subset N$ such that $a^{j} \in v\left(S^{j}\right)$ and $b>_{S^{j}} a^{j-1}$ for all $j=1, \ldots, p$.

(2) A subset $L$ of $A$ is said to be consistent if for all $x \in A$, $x \in L \Leftrightarrow$ for all $y \in A$, for all $S \subseteq N: y \in v(S), \exists z \in$ $L$ such that $[z=y$ or $z \gg y]$ and not $\left(z>_{S} x\right)$.

(3) The largest consistent set $\operatorname{LCS}(\Gamma)$ is the unique consistent set that includes any other consistent set. 
In order to compare the farsighted one-core and the largest consistent set, let us consider the following example due to Chwe [9, page 321].

Example 11. A committee game involves a set of players $N=$ $\{1,2,3\}$ and a set of outcomes $A=\{a, b, c, d, e\}$. Preferences which are linear are defined as follows: $\succ_{1}:$ cbeda, $\succ_{2}$ : dcabe, and $\succ_{3}$ : eabdc. The characteristic function $v$ is the majority rule defined by $v(S)=A$ if and only if $|S| \geq 2$.

According to the farsighted one-core the solution is $\{(1, c),(2, d),(3, e)\}$ and according to the largest consistent set, the solution is $\{(1, c),(2, d),(3, b)\}$ (since $\operatorname{LCS}(\Gamma)=\{b, c, d\}$ ).

With this Example 11, it is clear that neither the farsighted one-core includes the largest consistent set nor the largest consistent set includes the farsighted one-core. However, when preferences are linear orders, the result below highlights one advantage of the farsighted one-core over the largest consistent set.

Proposition 12. Let $\Gamma=\left(N, A, v,\left(>_{i}\right)_{i \in N}\right)$ where $|N|$ is odd and $v$ the majority rule. Then, for all $i \in N$, for all $x \in A$, $(i, x) \in \mathscr{F}(\Gamma) \Rightarrow x \geq_{i} y$ for all $y \in \operatorname{LCS}(\Gamma)$.

Proof. It is known that when preferences are linear orders, the set of not 1-dominated outcomes includes the largest consistent set (see Chwe [9, Proposition 7, page 321]). Moreover, for each $i$, the set $\widehat{S}^{i}$ includes the set of not 1-dominated outcomes. That is, $\operatorname{LCS}(\Gamma) \subset \widehat{S}^{i}(\Gamma)$ for all player $i$ and the result follows.

Nonemptiness of the Farsighted One-Core. It is well known that the Condorcet solution has a very strong stability requirement. As a consequence of this, it does not always exist.

As said above, the core of a committee game might be empty. If the core is nonempty, then $C^{i}$ is nonempty (implying that the one-core is nonempty) for each player $i$. Furthermore, any player $i$ prefers $y$ to $x$ whenever $x \in C^{i}$ and $y \in$ $\mathscr{C}_{1}(\Gamma)$. Shenoy [1] illustrated clearly the advantage of the onecore over the core through a simple example and proved that the one-core is nonempty for any $n$-person finite simple committee game when $n \leq 4$.

Although the behavioral pattern is improved in the onecore over the core, the problem of existence is still unsolved. Shenoy [1] provides in Example 4.2, page 393, the following 5 -player committee game with empty one-core.

Example 13. Consider $N=\{1,2,3,4,5\}, A=\left\{a_{0}, a_{1}, a_{2}, a_{3}\right.$, $\left.a_{4}, a_{5}\right\}$,

$$
v(S)=\left\{\begin{array}{l}
A \text { if }|S| \geq 3 \\
\emptyset \text { if }|S|<3,
\end{array}\right.
$$

and the payoff utility functions are shown in Table 1.

A calculation shows that $\widehat{S}^{i}=\left\{\left(i, a_{1}\right),\left(i, a_{2}\right),\left(i, a_{3}\right)\right.$, $\left.\left(i, a_{4}\right),\left(1, a_{5}\right)\right\}$ for each player $i \in N$. Then, $S^{1}=\left\{\left(1, a_{4}\right)\right\}$,
TABLE 1

\begin{tabular}{ccccccc}
\hline & $a_{0}$ & $a_{1}$ & $a_{2}$ & $a_{3}$ & $a_{4}$ & $a_{5}$ \\
\hline$u_{1}$ & 0 & 1 & 2 & 3 & 4 & 0 \\
$u_{2}$ & 0 & 2 & 3 & 4 & 0 & 1 \\
$u_{3}$ & 0 & 3 & 4 & 0 & 1 & 2 \\
$u_{4}$ & 0 & 4 & 0 & 1 & 2 & 3 \\
$u_{5}$ & 0 & 0 & 1 & 2 & 3 & 4 \\
\hline
\end{tabular}

$S^{2}=\left\{\left(2, a_{3}\right)\right\}, S^{3}=\left\{\left(3, a_{2}\right)\right\}, S^{4}=\left\{\left(4, a_{1}\right)\right\}$, and $S^{5}=\left\{\left(5, a_{5}\right)\right\}$. Hence, the farsighted one-core is given by $\mathscr{F}(\Gamma)=\left\{\left(1, a_{4}\right)\right.$, $\left.\left(2, a_{3}\right),\left(3, a_{2}\right),\left(4, a_{1}\right),\left(5, a_{5}\right)\right\}$.

Thus, the farsighted one-core is nonempty. We prove next that when indifference is not allowed in individual rankings or utilities vectors are componentwise different the farsighted one-core of every simple committee game is nonempty. But before that, we need the following result that proves the transitivity of the 1-dominance.

Proposition 14. The relation 1-dom is transitive over the set of simple committee games when individual preferences are linear orders. More precisely, for all $a, b, c \in A$, for all $S, T \in 2^{N}$, $\left[a 1-\operatorname{dom}_{S} b\right.$ and $\left.b 1-\operatorname{dom}_{T} c\right] \Longrightarrow a 1-\operatorname{dom}_{T} c$.

Proof. Consider three distinct alternatives $a, b, c$ and two winning coalitions $S$ and $T$ such that $a 1-\operatorname{dom}_{S} b$ and $b 1-$ $\operatorname{dom}_{T} c$. To show that $a 1-\operatorname{dom}_{T} c$ it suffices to prove that $a \operatorname{dom}_{T} c$ and for all $z \in A,\left(z \operatorname{dom} a \Rightarrow z \geq_{i} c\right.$ for all $i \in T)$.

Since $a 1-\operatorname{dom}_{S} b$ then $a \operatorname{dom}_{S} b$. In addition $b 1-\operatorname{dom}_{T} c$ and $a \operatorname{dom} b$ imply $a \geq_{i} c$ for all $i \in T$. That is, $a>_{i} c$ for all $i \in T$ since preferences are linear orders and this means that $a \operatorname{dom}_{T} c$, for the committee game, is simple. Next, let $z \in A$ such that $z$ dom $a$. Since $a 1-\operatorname{dom}_{S} b$ then, for each $i \in S, z \geq_{i} b$. That is, $z \operatorname{dom}_{S} b$. Moreover, $b 1-\operatorname{dom}_{T} c$. Thus, for each $i \in T, z \geq_{i} c$; that is, for all $i \in T, z \succ_{i} c$, or $z \operatorname{dom}_{T} c$ since preferences are linear orders.

Now we prove the main result of this section that deals with the nonemptiness of the farsighted one-core of any simple committee game in which preferences are linear orders and the nonemptiness of the farsighted one-core of any 3-player committee game.

Proposition 15. For any simple committee game $\Gamma$ where preferences are linear orders, $\mathscr{F}(\Gamma) \neq \emptyset$.

Proof. Let $\Gamma=\left(N, A, v,\left(\succ_{i}\right)_{i \in N}\right)$; since $\mathscr{F}(\Gamma)=\bigcup_{i \in N} S^{i}$, it is enough to prove that for all $i \in N, S^{i} \neq \emptyset$. On the other hand, by definition of $S^{i}=\left\{(i, x) \in \widehat{S}^{i}: x \succeq_{i} y\right.$ for all $\left.(i, y) \in \widehat{S}^{i}\right\}$, again it is enough to prove that $\widehat{S}^{i} \neq \emptyset$. Assume that for some player $i \in N, \widehat{S}^{i}=\emptyset$. Then there exists a sequence $\left(S_{k}\right)_{0 \leq k \leq p}$ of coalitions with $i \notin S_{k}$ for each $0 \leq k \leq p$ and a sequence $\left(a_{k}\right)_{0 \leq k \leq p}$ of candidates such that $a_{k} 1-\operatorname{dom}_{s_{k}} a_{k-1}$ for each $k=$ $1, \ldots, p$ and $a_{0} 1-\operatorname{dom}_{S_{0}} a_{p}$. 
We have $a_{2} 1-\operatorname{dom}_{S_{2}} a_{1}$ and $a_{1} 1-\operatorname{dom}_{S_{1}} a_{0}$, which imply by transitivity of the relation 1-dom that $a_{2} 1$-dom $\mathcal{S}_{1} a_{0}$. By the same way, we can show that for each $k=1, \ldots, p$ and $a_{k} 1-$ dom $_{S_{1}} a_{0}$, in particular, $a_{p} 1-\operatorname{dom}_{S_{1}} a_{0}$. Thus, $a_{0} 1-\operatorname{dom}_{S_{0}} a_{p}$ and $a_{p} 1-\operatorname{dom}_{S_{1}} a_{0}$. Since the committee game is simple and $a_{0} \in v\left(S_{0}\right), a_{p} \in v\left(S_{1}\right)$ we have $v\left(S_{0}\right)=A=v\left(S_{1}\right)$. This implies by condition $\left(C_{3}\right)$ that $S_{0} \cap S_{1} \neq \emptyset$. Let $i_{0} \in$ $S_{0} \cap S_{1}$; then the following holds: $a_{0}>_{i_{0}} a_{p}>_{i_{0}} a_{0}$ and this is a contradiction.

For general committee games (that need not be simple) we prove that if the number of players is three, then the farsighted one-core is nonempty.

Proposition 16. For any 3-player committee game $\Gamma$, $\mathscr{F}(\Gamma) \neq \emptyset$.

Proof. Let $\Gamma=\left(N, A, v,\left(\succcurlyeq_{i}\right)_{i \in N}\right)$ be a 3-player committee game. Since $\mathscr{F}(\Gamma)=\bigcup_{i \in N} S^{i}$, it is enough to prove that for all $i \in N, \widehat{S}^{i} \neq \emptyset$. Assume that for some player $i \in N, \widehat{S}^{i}=\emptyset$. Then there exists a sequence $\left(S_{k}\right)_{0 \leq k \leq m}$ of coalitions with $i \notin S_{k}$ for each $0 \leq k \leq m$ and a sequence $\left(a_{k}\right)_{0 \leq k \leq m}$ of distinct candidates such that $a_{k} 1-\operatorname{dom}_{S_{k}} a_{k-1}$ for each $k=1, \ldots, m$ and $a_{0}$ 1-dom do $_{0} a_{m}$.

Since $a_{p} \neq a_{q}$ for each $p, q \in\{0, \ldots, m\}$ with $p \neq q$, then $\emptyset \neq v\left(S_{p}\right) \neq v\left(S_{q}\right) \neq \emptyset$, which implies $S_{p} \cap S_{q} \neq \emptyset$ for each $p, q \in$ $\{0, \ldots, m\}$ with $p \neq q$.

(i) If for each $p \in\{0, \ldots, m\}\left|S_{p}\right|=2$, then $S_{p}=$ $N \backslash\{i\}$. Then, the following holds for some player $i_{0}: a_{0}>_{i_{0}} a_{m}>_{i_{0}} a_{m-1}>_{i_{0}} \cdots>_{i_{0}} a_{2}>_{i_{0}} a_{1}>_{i_{0}} a_{0}$ which is a contradiction.

(ii) If not, there exists $p_{0} \in\{0, \ldots, m\}$ such that $\left|S_{p_{0}}\right|=$ 1. Let $S_{p_{0}}=\{j\}$ : since $S_{p} \cap S_{q} \neq \emptyset$ for each $p, q \in$ $\{0, \ldots, m\}$ with $p \neq q$; then $S_{p_{0}} \cap S_{q} \neq \emptyset$ for each $q \in$ $\{0, \ldots, m\}$ with $p_{0} \neq q$. Therefore, $j \in S_{q}$ for each $q \in\{0, \ldots, m\}$. For that player $j$, we have the following: $a_{0}>_{j} a_{m}>_{j} a_{m-1}>_{j} \cdots>_{j} a_{2}>_{j} a_{1}>_{j} a_{0}$ which is also a contradiction.

\section{The Bargaining Set Revised}

Shenoy [1] defines a bargaining set that is relevant to the context of a committee game and is presented as an extension of the one-core. It is based, as all the well-known references on bargaining set in the context of games with and without side payments (Aumann and Maschler [3], Peleg [4], Billera [5, 6], D'Aspremont [7], Asscher [8], and others), on objections and counter-objections but is quite different from all of them. An objection against proposal $(i, x)$ is a triple $(j, S, y)$ such that $j \in S \in 2^{N}, y \in A, i \notin S$, and $y \operatorname{dom}_{S} x$. The rationale behind the dominance is that a coalition $S$ such that $y \in$ $v(S)$ and members of which prefer $y$ to $x$ should vote for $y$ against $x$ whenever they are given the possibility to. But as argued before, this is a myopic behavior. Our purpose is to explore the consequence of replacing the dominance with the more foresight dominance, the 1-dominance in the definition of objection on one hand and on the definition of counterobjection on the other hand.
Definition 17. Let $\Gamma=\left(N, A, v,\left(\succeq_{i}\right)_{i \in N}\right)$ be a committee game.

(1) An $R$-objection against a proposal $(i, x)$ is a triple $(j, S, y)$ such that

$$
\begin{gathered}
j \in S \in 2^{N}, \quad y \in A \backslash\{x\}, \\
i \notin S, \quad y 1-\operatorname{dom}_{S} x .
\end{gathered}
$$

(2) An $R$-counter-objection against objection $(j, S, y)$ to $(i, x)$ is a triple $(k, T, z)$, where

$$
\begin{gathered}
k \in T \in 2^{N}, \quad z \in A \backslash\{x, y\}, \\
\text { either } k=i \text { or } x \succ_{k} y, \\
z 1-\operatorname{dom}_{T} y, \quad z \geq_{k} x, \\
\exists e \in A: e \operatorname{dom} z, \quad x \succ_{j} e .
\end{gathered}
$$

(3) A proposal is said to be $R M$-stable if every $R$ objection has an $R$-counter-objection. Let $R \widehat{M}$ be the set of all $R M$-stable proposals. An $R M$-stable proposal $(i, a)$ is said to be maximal if $a \geq_{i} x$ for all $x$ such that $(i, x) \in R \widehat{M}$.

(4) The farsighted bargaining set $\mathscr{F} \mathscr{M}$ is the set of all maximal $R M$-stable proposals.

Below, we recall the Shenoy [1] definition of the bargaining set.

Definition 18. Let $\Gamma=\left(N, A, v,\left(\succeq_{i}\right)_{i \in N}\right)$ be a committee game.

An objection against a proposal $(i, x)$ is a triple $(j, S, y)$ such that

$$
\begin{gathered}
j \in S \in 2^{N}, \quad y \in A \backslash\{x\}, \\
i \notin S, \quad y \operatorname{dom}_{S} x .
\end{gathered}
$$

A counter-objection against objection $(j, S, y)$ to $(i, x)$ is a triple $(k, T, z)$ such that

$$
\begin{array}{r}
k \in T \in 2^{N}, \quad z \in A \backslash\{x, y\}, \\
\text { either } k=i \quad \text { or } x \succ_{k} y, \\
z \operatorname{dom}_{T} y, \quad x \succ_{j} z, \quad z \succeq_{k} x .
\end{array}
$$

If $(j, S, y)$ is an objection against a proposal $(i, x)$, player $j$ expects the players in coalition $S$ to vote for $y$ which would result in $y$ winning against $x$. The counter-objection which is a reply by player $k$ is made either by player $i$ or by a player who stands to lose if the objection is carried out. If a counterobjection does exist, there is a strong motivation for player $j$ to withdraw his objection. On the other hand, if there is no counter-objection, then player $j$ has a justified objection and player $i$ cannot expect to get his proposal accepted by the committee.

Definition 19. (1) A proposal is said to be $M$-stable if every objection has a counter-objection. Let $\widehat{M}$ be the set of all 
$M$-stable proposals. A $M$-stable proposal $(i, a)$ is said to be maximal if $a \geq_{i} x$ for all $x$ such that $(i, x) \in \widehat{M}$.

(2) The bargaining set $\mathscr{U}$ is the set of all maximal $M$ stable proposals.

First, let us reconsider Example 6 and compute the bargaining set and the farsighted bargaining set. A simple calculation shows that the set of all $R M$-stable proposals $R \widehat{M}(\Gamma)$ is given by $R \widehat{M}(\Gamma)=\{(1, a),(1, b),(1, c),(1, d),(2, a)$, $(2, b),(2, d),(3, a),(3, b),(3, c),(3, d)\}$.

Hence, $\mathscr{F} \mathscr{M}(\Gamma)=\{(1, b),(2, b),(3, d)\}$ while $\mathscr{M}(\Gamma)=$ $\{(1, a),(2, b),(3, d)\}$.

Again, we can see that the farsighted bargaining set makes a better prediction. While for the bargaining set player 1 should propose $a$, with respect to the farsighted bargaining set, he should rather propose $b$, which is better in his point of view.

We now show that the farsighted bargaining set can be nonempty while the Shenoy bargaining set is empty.

Proposition 20. There exists a committee game $\Gamma$ for which the one-core $\mathscr{C}_{1}(\Gamma)$ and the bargaining set $\mathscr{M}(\Gamma)$ are empty meanwhile the farsighted bargaining set $\mathscr{F} \mathscr{M}(\Gamma)$ is nonempty.

Proof. Consider the committee game defined in Example 13. For that game, $\mathscr{C}_{1}(\Gamma)$ and the bargaining set $\mathscr{M}(\Gamma)$ are empty. Meanwhile the farsighted bargaining set $\mathscr{F} \mathscr{M}(\Gamma)$ is nonempty and the computation gives $\mathscr{F} \mathscr{M}(\Gamma)=$ $\left\{\left(1, a_{4}\right),\left(2, a_{3}\right),\left(3, a_{2}\right),\left(4, a_{1}\right),\left(5, a_{5}\right)\right\}$.

Indeed, the set of not 1-dominated outcomes of this game is $A \backslash\left\{a_{0}\right\}$. Thus, for each $i \in\{1,2,3,4,5\}$, the proposal $(i, x)$ with $x \in A \backslash\left\{a_{0}\right\}$ is $R M$-stable and since the farsighted bargaining set is the set of all maximal $R M$-stable proposals, then the result holds.

Shenoy [1] shows that, if the Condorcet solution, say $\alpha$, exists, the Shenoy bargaining set is given by $\mathscr{M}(\Gamma)=$ $\{(1, \alpha) ;(2, \alpha) ; \ldots ;(n, \alpha)\}$. We prove below that this result also holds for the farsighted bargaining set.

Proposition 21. Let $\Gamma$ be a committee game such that the Condorcet solution, say $\alpha$, exists. Then, the farsighted bargaining set is given by $\mathscr{F} \mathscr{M}(\Gamma)=\{(1, \alpha),(2, \alpha), \ldots,(n, \alpha)\}$.

Proof. Obviously, $(i, \alpha) \in \mathscr{F} \mathscr{M}(\Gamma)$ for each player $i \in N$.

Assume that there is a proposal $(i, x)$ of some player $i$ such that $(i, x) \in \mathscr{F} \mathscr{M}(\Gamma)$ with $x \neq \alpha$. Then, $x \sim_{i} \alpha$. But $\alpha$ being a condorcet solution implies $\alpha$ dom $x$ via a certain coalition $S$ and $\alpha$ is undominated, that is, $\alpha 1-\operatorname{dom}_{S} x$, and since $x \sim{ }_{i} \alpha$, then $i \notin S$. Hence, for a player $j \in S,(j, S, \alpha)$ is an $R$-objection to proposal $(i, x)$ that does not have a $R$-counter-objection since $\alpha$ is undominated. Therefore, $(i, x) \notin \mathscr{F} \mathscr{M}(\Gamma)$ which is a contradiction: hence, $\mathscr{F} \mathscr{M}(\Gamma)=$ $\{(1, \alpha),(2, \alpha), \ldots,(n, \alpha)\}$.

Farsighted One-Core and Shenoy Bargaining Set. The definition of the farsighted one-core and of the Shenoy bargaining set seems a bit similar at a first sight, but these notions differ significantly. The following result states that each player by proposing his proposals from the farsighted one-core does as good (if not better) as any outcome in the Shenoy bargaining set.

Proposition 22. Let $\Gamma=\left(N, A, v,\left(\succeq_{i}\right)_{i \in N}\right)$ be a committee game. Then, for all $i \in N$, for all $x \in A,(i, x) \in \mathscr{F}(\Gamma) \Rightarrow x \geq_{i} y$ for all $y$ such that $(i, y) \in \mathscr{M}(\Gamma)$.

Proof. Assume that there exists, for some player $i_{0}$, a proposal $\left(i_{0}, x\right) \in \mathscr{F}(\Gamma)$ and another proposal $\left(i_{0}, y\right) \in \mathscr{M}(\Gamma)$ with $y>_{i_{0}} x$. Since $\left(i_{0}, y\right) \in \mathscr{M}(\Gamma)$ then $\left(i_{0}, y\right)$ is $R M$-stable. That is, either there is no objection to $\left(i_{0}, y\right)$ or each objection to $\left(i_{0}, y\right)$ has a counter-objection.

(i) If there is no objection to $\left(i_{0}, y\right)$ then $y$ is undominated or, for each $S$ such that $y$ is dominated via $S$, $i_{0} \in S$ and $y$ is undominated via $S \backslash\left\{i_{0}\right\}$. Thus, $\left(i_{0}, y\right) \in$ $\widehat{C}^{i_{0}}$ with $y \succ_{i_{0}} x$, which means that $\left(i_{0}, y\right) \in \mathscr{F}(\Gamma)$ and $\left(i_{0}, x\right) \notin \mathscr{F}(\Gamma)$. This is a contradiction.

(ii) If there exists an objection to $\left(i_{0}, y\right)$, let $(j, S, a)$ be such an objection that admits a counter-objection $(k, T, z)$. Then $i_{0} \notin S, a \operatorname{dom}_{S} y$ and $z \operatorname{dom}_{T} a$. But from the definition of the counter-objection we have $y \succ_{j} z$. Thus, non $\left(z \succeq_{i} y\right)$ for each player $i \in$ $S$. Therefore, $\operatorname{non}\left(a 1-\operatorname{dom}_{S} y\right)$ which means that $\left(i_{0}, y\right) \in \widehat{S}^{i_{0}}$. Since $\left(i_{0}, x\right) \in \mathscr{F}(\Gamma)$ we have $x \geq_{i_{0}} y$ which contradicts the assumption $y \succ_{i_{0}} x$.

Proposition 23. (1) For all committee game $\Gamma=(N, A, v$, $\left.\left(\succeq_{i}\right)_{i \in N}\right), \widehat{M}(\Gamma) \subset \bigcup_{i \in N} \widehat{S}^{i}(\Gamma)$.

(2) The above inclusion may be strict.

Proof. (1) Let $(i, x)$ be an $M$-stable proposal. Either there is no objection or each objection has a counter-objection. If there is no objection to $(i, x)$, then $(i, x)$ is undominated and therefore is not 1-dominated. If not, let $(j, S, y)$ be an objection and $(k, T, z)$ a counter-objection. We have $x>_{j} z$, with $y \operatorname{dom}_{s} x$, $z$ dom $y$, and $j \in S$. That is, $(i, x)$ is not 1-dominated.

(2) In the Shenoy [1] Example 4.2, recalled here in Example 13, the bargaining set is empty and the farsighted one-core is nonempty.

Farsighted One-Core and Farsighted Bargaining Set. The following result deals with the comparison of the farsighted onecore and the farsighted bargaining set.

Proposition 24. (1) For each committee game $\Gamma$ where preferences are linear orders, $\mathscr{F} \mathscr{M}(\Gamma)=\mathscr{F}(\Gamma)$.

(2) There exists committee games such that $\mathscr{F} \mathscr{M}(\Gamma) \neq \mathscr{F}(\Gamma)$.

Proof. (1) Let $\Gamma$ be a committee game. Assume that for all $i, \geq_{i}$ is a linear order. According to Proposition 15, the farsighted one-core $\mathscr{F}(\Gamma)$ is nonempty. It follows that $\emptyset \neq \bigcup_{i \in N} \widehat{S}^{i} \subset R \widehat{M}$, which implies $\mathscr{F}(\Gamma) \subset \mathscr{F} \mathscr{M}(\Gamma)$.

Conversely, let $(i, x) \in \mathscr{F} \mathscr{M}(\Gamma)$ and assume there exists an $R$-objection $(j, S, y)$ against $(i, x)$ that has an $R$-counter-objection $(k, T, z)$. Then the following holds: 


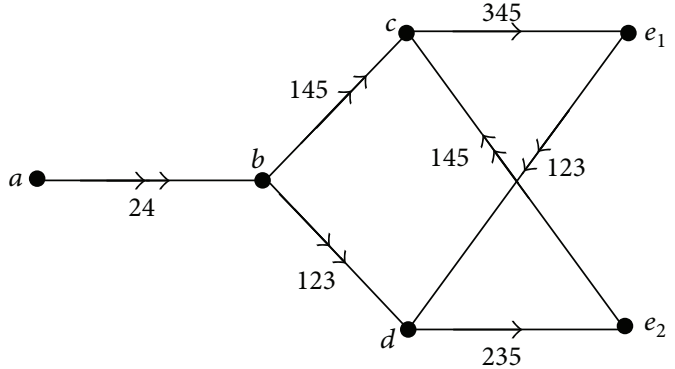

ऋ-dominance relation

$\rightarrow$ Classical dominance relation

Figure 2: $\mathscr{F} \mathscr{M}(\Gamma)$ and $\mathscr{F}(\Gamma)$ are different.

$z 1-\operatorname{dom}_{T} y, y 1-\operatorname{dom}_{S} x$ and by the transitivity of 1-dom, we have $z 1-\operatorname{dom}_{S} x$. Since $(k, T, z)$ is an $R$-counter-objection, there exists $e \in A: e \operatorname{dom} z$ and $x \succ_{j} e$. Since $z 1-\operatorname{dom}_{S} x$, $e \operatorname{dom} z$, and $j \in S$, it follows that $e \succeq_{j} x$ which contradicts $x>{ }_{j} e$. Therefore there is no objection to any proposal $(i, x) \in$ $\mathscr{F} \mathscr{M}(\Gamma)$, meaning that $\mathscr{F} \mathscr{M}(\Gamma) \subset \mathscr{F}(\Gamma)$.

(2) Consider the following committee game that involves a set of players $N=\{1,2,3,4,5\}$ and a set of outcomes $A=\left\{a, b, c, d, e_{1}, e_{2}\right\}$. Preferences are as follows: $\succeq_{1}:(a d c)\left(e_{1} e_{2}\right) b, \geq_{2}: e_{2} d b(a c) e_{1}, \geq_{3}: e_{2}(a d)\left(b e_{1}\right) c, \geq_{4}: e_{1} c b$ $(d a) e_{2}$, and $\geq_{5}: e_{1}(a c)\left(b e_{2}\right) d$. The characteristic function $v$ is defined by $v(24)=\{b\}, v(145)=\{c\}, v(123)=\{d\}$, $v(345)=\left\{e_{1}\right\}, v(235)=\left\{e_{2}\right\}, v(S)=\{b\}$ if $|S|=3$ and $24 \subset S$, $v(S)=A$ if $|S| \geq 4$ and $v(S)=\emptyset$ elsewhere. The computation of the dominance graph gives, as shown in Figure 2, the following.

One can observe on one hand that $(1, a) \notin \mathscr{F}(\Gamma)$. On the other hand, $(1, a) \in \mathscr{F} \mathscr{M}(\Gamma)$. Indeed, the only $R$-objections to proposal $(1, a)$ that can be made by players are $(2,24, b)$ and $(4,24, b)$ by players 2 and 4 , respectively.

If we consider $(2,24, b)$, then $(1,145, c)$ is an $R$-counterobjection since $c 1-\operatorname{dom}_{145} b, a \succ_{1} b, c \geq_{1} a$, and $e_{1} \operatorname{dom} c$ with $a>_{2} e_{1}$.

As well consider $(4,24, b)$; then $(1,123, d)$ is an $R$ counter-objection since $d 1-\operatorname{dom}_{123} b, a \succ_{1} b, d \geq_{1} a$, and $e_{2}$ dom $d$ with $a>_{4} e_{2}$.

Thus, proposal $(1, a)$ is $R M$-stable and, since $a$ is maximal with respect to player 1 preference, it follows that $(1, a) \in$ $\mathscr{F} \mathscr{M}(\Gamma)$.

The following result shows the advantage of the farsighted bargaining set over the farsighted one-core.

Proposition 25. Let $\Gamma=\left(N, A, v,\left(\succeq_{i}\right)_{i \in N}\right)$ be a committee game. Then, for all $i \in N$, for all $x \in A,(i, x) \in \mathscr{F} \mathscr{M}(\Gamma) \Rightarrow$ $x \geq_{i} y$ for all $y$ such that $(i, y) \in \mathscr{F}(\Gamma)$.

Proof. Let $\Gamma$ be a committee game. Let $(i, x) \in \mathscr{F} \mathscr{M}(\Gamma)$ and $(i, y) \in \mathscr{F}(\Gamma)$. Since $(i, y) \in \mathscr{F}(\Gamma)$, there is no $R$-objection to proposal $(i, y)$. Therefore, $(i, y)$ is an $R M$-stable proposal. Moreover, $(i, x) \in \mathscr{F} \mathscr{M}(\Gamma)$ means $(i, x)$ is a maximal $R M$ stable proposal. Hence, $x \geq_{i} y$.
Nonemptiness of the Farsighted Bargaining Set. Shenoy [1] proved that if $\Gamma$ is a finite committee game with a nonempty core, then

(i) the Shenoy bargaining set $\mathscr{M}(\Gamma)$ is nonempty;

(ii) for any player $i$, there exists an option $x$ such that $(i, x) \in \mathscr{M}(\Gamma)$;

(iii) any player $i$ is better off at any $x$ than at $y$ where $(i, x) \in$ $\mathscr{M}(\Gamma)$ and $y \in C(\Gamma)$.

The above three items still hold with the one-core.

In general committee games, the nonemptiness of the Shenoy bargaining set ensures the nonemptiness of the farsighted bargaining set as we can observe in the result below.

Proposition 26. Let $\Gamma$ be a committee game such that $\mathscr{M}(\Gamma) \neq \emptyset$; then $\mathscr{F} \mathscr{M}(\Gamma) \neq \emptyset$.

Proof. Let $\Gamma$ be a committee game. Assume that $\mathscr{M}(\Gamma)$ is nonempty. Let $(i, x) \in \mathscr{M}(\Gamma)$; then, $(i, x) \in R \widehat{M}(\Gamma)$. Indeed, $(i, x) \in \mathscr{M}(\Gamma)$ means that either there is no objection to $(i, x)$ or any objection to $(i, x)$ admits a counter-objection.

(i) If there is no objection to $(i, x)$ then $(i, x)$ is a core proposal or $(i, x) \in \widehat{C}^{i_{0}} \subseteq \widehat{S}^{i_{0}} \subseteq R \widehat{M}(\Gamma)$.

(ii) Assume that there exists an objection to $(i, x)$. Let $(j, S, y)$ be an $R$-objection to $(i, x)$. Then, $y 1$ $\operatorname{dom}_{S} x$ with $i \notin S$, which implies $y \operatorname{dom}_{S} x$ with $i \notin S$. Thus, $(j, S, y)$ is an objection to proposal $(i, x)$. From the assumption that any objection has a counter-objection, let $(k, T, z)$ be a counter-objection to $(j, S, y)$. From the definition of the counterobjection we have $z \operatorname{dom}_{T} y$ with $x>_{j} z$. This implies not $\left(y 1-\operatorname{dom}_{S} x\right)$ which contradicts the fact that $(j, S, y)$ is an $R$-objection to $(i, x)$. Hence, there is no $R$-objection to $(i, x)$ and, therefore, $(i, x) \in$ $R \widehat{M}(\Gamma)$. In all cases, $R \widehat{M}(\Gamma)$ is nonempty; hence $\mathscr{F} \mathscr{M}(\Gamma) \neq \emptyset$.

If the committee game is simple and preferences are linear orders, it follows from Propositions 15 and 24 that the farsighted bargaining set is nonemptiness.

Proposition 27. For any simple committee game $\Gamma$ where preferences are linear orders, $\mathscr{F} \mathscr{M}(\Gamma) \neq \emptyset$.

For general (not necessarily simple) committee games, we have the following proposition stating the nonemptiness of the farsighted bargaining set (as well as the Shenoy bargaining set) of any 3-player committee game.

Proposition 28. For any 3-player committee game $\Gamma$, $\mathscr{F} \mathscr{M}(\Gamma) \neq \emptyset$.

Proof. Let $\Gamma$ be a 3-player committee game. According to Proposition 16, $\mathscr{F}(\Gamma)$ is nonempty. Let $(i, x) \in \mathscr{F}(\Gamma)$; then, there is no $R$-objection to $(i, x)$ since it is not 1-dominated. Hence $(i, x) \in R \widehat{M}(\Gamma)$ and the conclusion follows. 


\section{Conclusion}

We came back on the four different solution concepts studied in relation to committee games by Shenoy [1] with emphasis on the one-core and the bargaining set. After showing that the one-core is subject to a lack of foresight, we introduce another solution concept, the farsighted one-core which is better than the one-core in terms of behavioral standard, which is always nonempty when individual preferences are linear orders, a property not shared neither by the one-core nor by the Shenoy bargaining set. This latter set has also been revised and we introduce a new bargaining set which is also better than the latter for committee games and is always nonempty when restricted to simple committee games in which preferences are strict orders.

We prove furthermore that any 3-player committee game has a nonempty farsighted one-core and we did not succeed in giving a characterization of general committee games inducing a nonempty farsighted one-core. Although the emptiness of the farsighted bargaining set of a simple committee game is guaranteed, the general study of the nonemptiness of this Shenoy revised solution concept is still an open problem.

\section{Conflict of Interests}

The authors declare that there is no conflict of interests regarding the publication of this paper.

\section{Acknowledgments}

The authors are very indebted to the anonymous referees for their insightful comments and suggestions which contributed to improvement of the presentation of this paper. The members of the MASS Laboratory of The University of Yaounde 1 are acknowledged for their comments and advices.

\section{References}

[1] P. P. Shenoy, "On committee decision making: a game theoretical approach," Management Science, vol. 26, no. 4, pp. 387-400, 1980.

[2] J. von Neumann and O. Morgenstern, Theory of Games and Economic Behavior, vol. 1953, Princeton University Press, Princeton, NJ, USA, 3rd edition, 1944.

[3] R. J. Aumann and M. Maschler, "The bargaining set for cooperative games," in Advances in Game Theory, M. Dresher, L. S. Shapley, and A. W. Tucker, Eds., vol. 52 of Annals of Mathematics Studies, pp. 443-476, Princeton University Press, Princeton, NJ, USA, 1964.

[4] B. Peleg, "Bargaining sets of cooperative games without side payments," Israel Journal of Mathematics, vol. 1, pp. 197-200, 1963.

[5] L. J. Billera, On cores and bargaining sets for $\mathrm{n}$-person cooperative games without side payments [Ph.D. dissertation], Department of Mathematics, The City University of New York, New York, NY, USA, 1968.

[6] L. J. Billera, "Existence of general bargaining sets for cooperative games without side payments," Bulletin of the American Mathematical Society, vol. 76, pp. 375-379, 1970.
[7] C. L. D'Aspremont, The bargaining set concept for cooperative games without side payments [Ph.D. dissertation], Stanford University, 1973.

[8] N. Asscher, "An ordinal bargaining set for games without side payments," Mathematics of Operations Research, vol. 1, no. 4, pp. 381-389, 1976.

[9] M. S.-Y. Chwe, "Farsighted coalitional stability," Journal of Economic Theory, vol. 63, no. 2, pp. 299-325, 1994.

[10] L. Xue, "Nonemptiness of the largest consistent set," Journal of Economic Theory, vol. 73, no. 2, pp. 453-459, 1997.

[11] L. Xue, "Coalitional stability under perfect foresight," Economic Theory, vol. 11, no. 3, pp. 603-627, 1998.

[12] A. Suzuki and S. Muto, "Farsighted stability in an $n$-person prisoner's dilemma," International Journal of Game Theory, vol. 33, no. 3, pp. 431-445, 2005.

[13] S. Béal, J. Durieu, and P. Solal, "Farsighted coalitional stability in TU-games," Mathematical Social Sciences, vol. 56, no. 3, pp. 303-313, 2008.

[14] J. A. M. Kenfack and B. Tchantcho, Farsighted Stability in TUGames with Endogenous Partition Structure, mimeo, 2013.

[15] A. Rubinstein, "Stability of decision systems under majority rule," Journal of Economic Theory, vol. 23, no. 2, pp. 150-159, 1980.

[16] D. B. Gillies, "Solutions to general non-zero-sum games," in Contributions to the Theory of Games, Volume IV, A. W. Tucker and R. D. Luce, Eds., vol. 40 of Annals of Mathematics Studies, pp. 47-85, Princeton University Press, Princeton, NJ, USA, 1959.

[17] L. S. Shapley, "Simple games: an outline of the descriptive theory," Behavioral Science, vol. 7, pp. 59-66, 1962.

[18] M. A. J. Condorcet, Essai sur l'Application de l'Analyse à la Probabilité des Décisions Rendues à la Pluralité des Voix, Imprimerie Royale, Paris, France, 1785.

[19] C. L. Dodgson, A Discussion of the Various Methods of Procedure in Conducting Elections, 1873, Reprinted in D. Black, The Theory of Committees and Elections, Cambridge University Press, Cambridge, UK, 1958. 


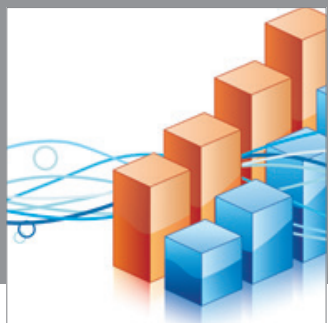

Advances in

Operations Research

mansans

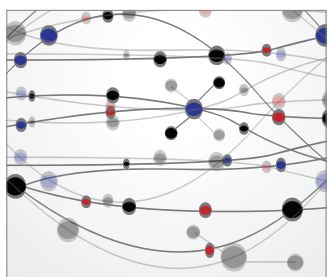

The Scientific World Journal
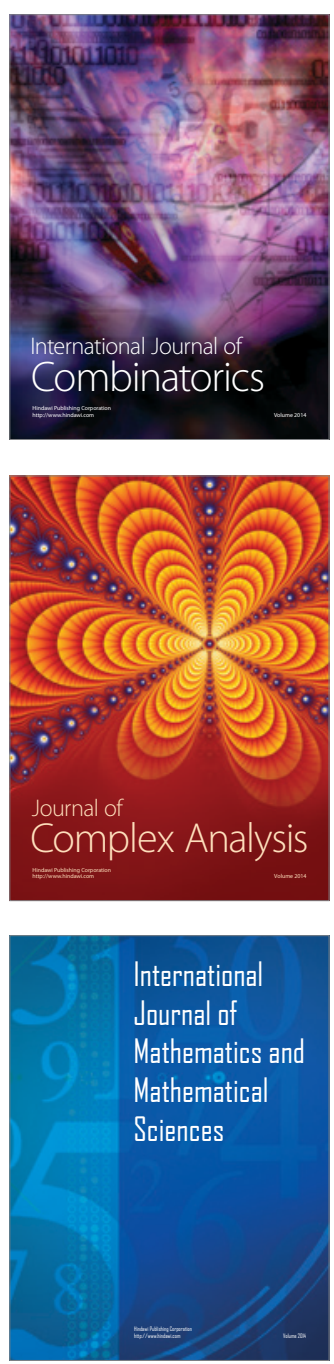
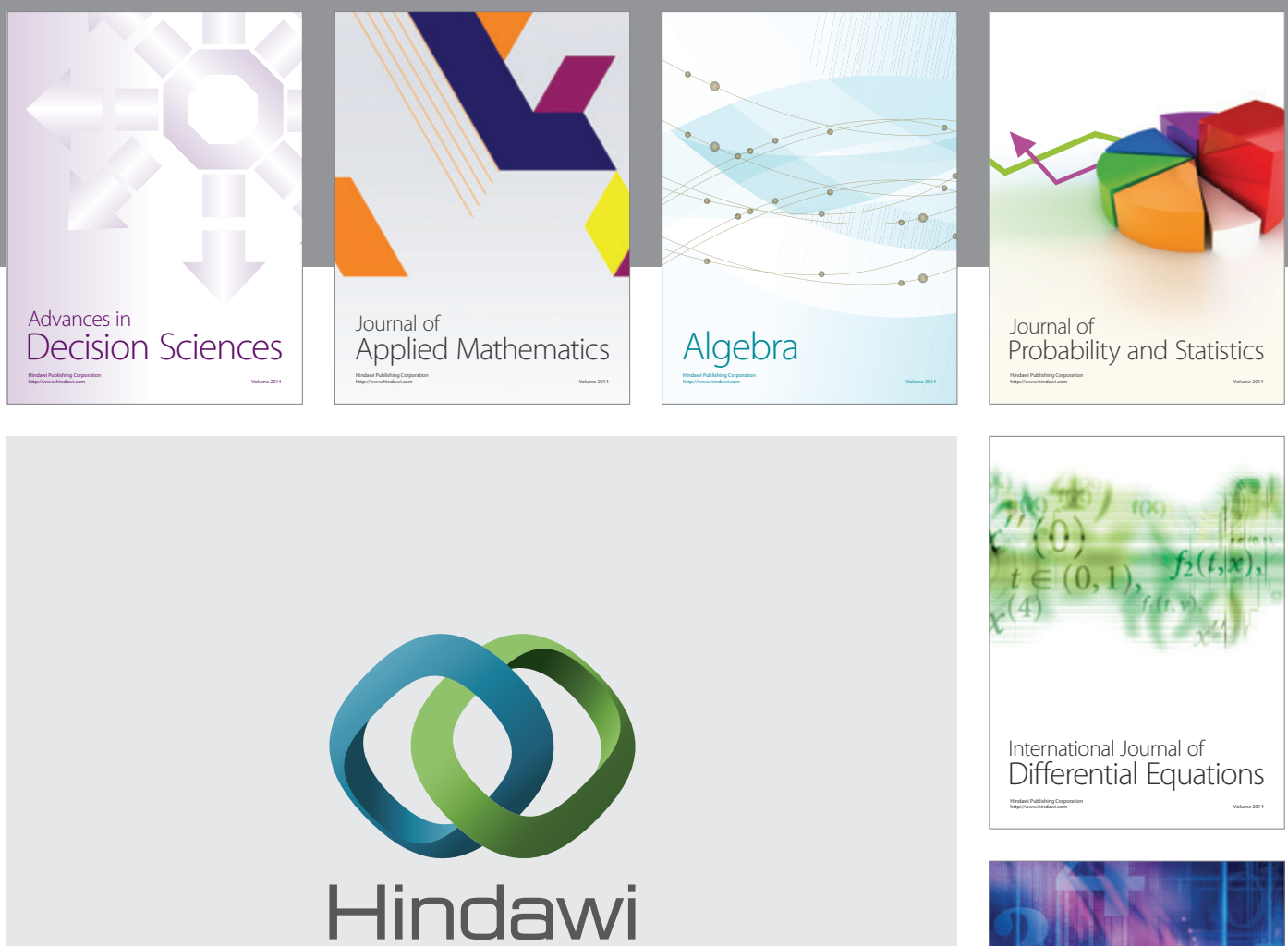

Submit your manuscripts at http://www.hindawi.com
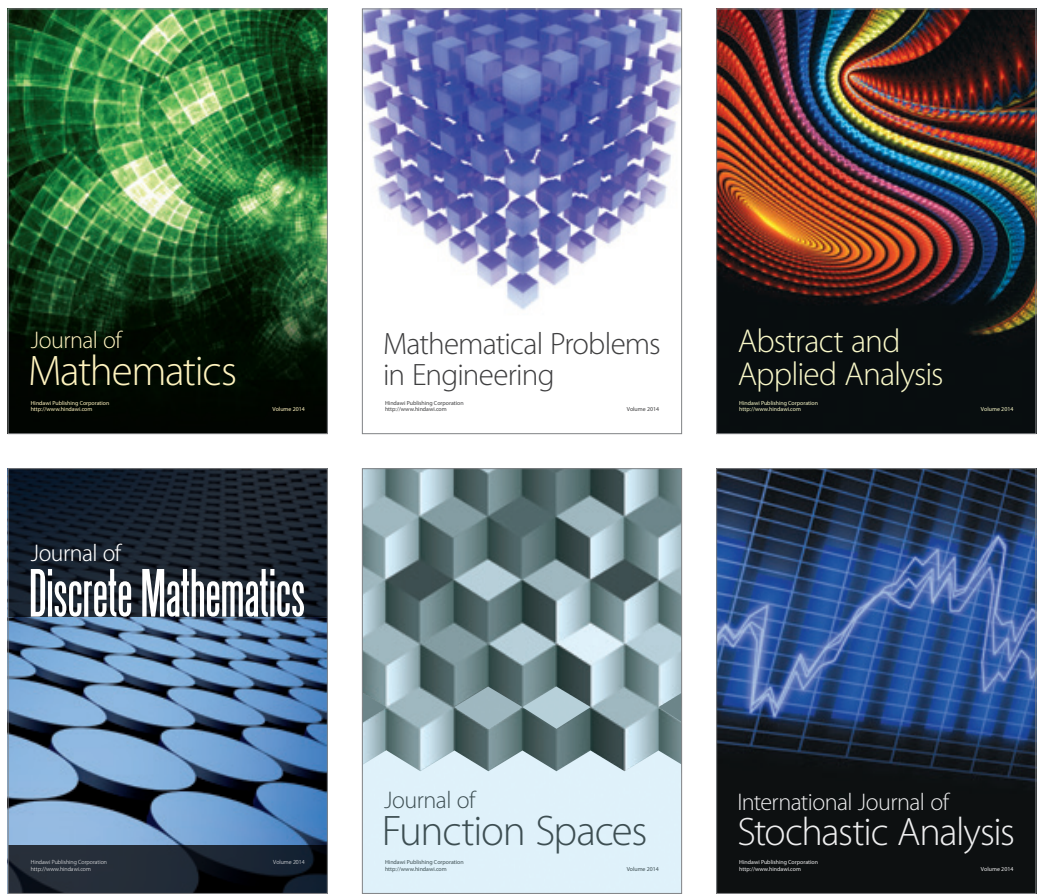

Journal of

Function Spaces

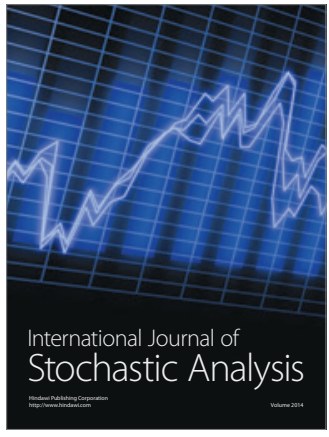

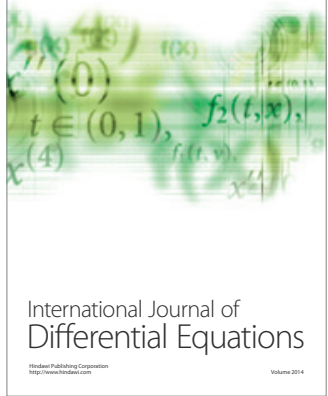
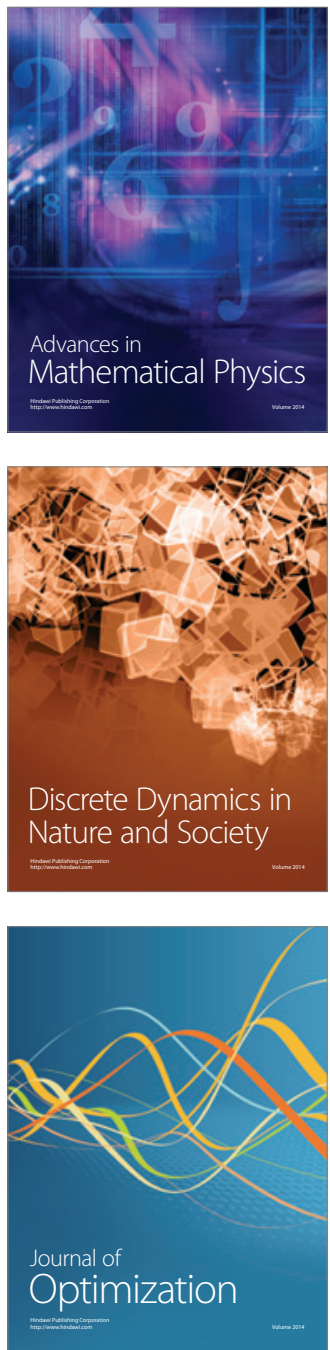